\title{
Vitamin D levels and SARS-CoV-2 assay results in health care workers in Brazil
}

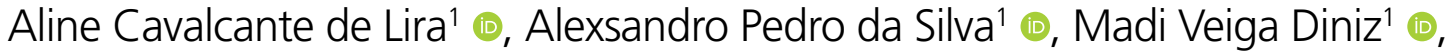 \\ Arione Vieira do Nascimento ${ }^{1} \odot$, Wagner Roberto Cirilo da Silva ${ }^{2} \odot$, Caroline Louise Diniz Pereira ${ }^{3} \odot$,

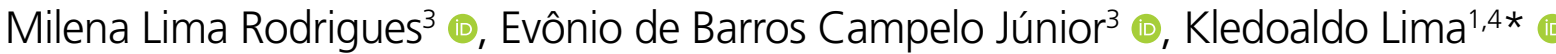

\section{INTRODUCTION}

A new coronavirus, which emerged in China in December 2019 (severe acute respiratory syndrome coronavirus 2, SARS$\mathrm{CoV}-2$ ), has led to a severe acute respiratory syndrome (coronavirus disease 2019, COVID-19) whose severity of symptoms has been linked to different biological risk factors, such as diabetes, hypertension, cardiovascular diseases, cancer, and cerebrovascular diseases ${ }^{1}$.

Although Hastie et al. did not confirm the association between lower plasma vitamin $\mathrm{D}$ rules and the risk of SARSCoV-2 infection, several other studies associated vitamin D with less clinical severity and positive testing for COVID-19

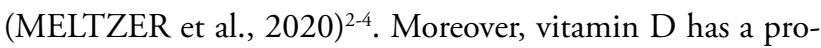
tective effect on the alveolar epithelium and reduces damage to the alveolar capillaries, preventing severe acute respiratory syndrome (SRAG) ${ }^{5}$.

In addition to COVID-19 having a lower incidence in children and pregnant women, several studies have demonstrated protective effects, less inflammation and an association of higher concentrations of vitamin $\mathrm{D}$, and lesser chances of infection by SARS-CoV-2 ${ }^{3,4,6}$. To our knowledge, there are no reports of this type of study in Brazil nor of the assessment of serum levels of vitamin $\mathrm{D}$ in health care professionals who work with patients or clinicians diagnosed with COVID-19. This study aimed to evaluate the serum concentration of vitamin $\mathrm{D}$ in health care professionals who work directly with patients or their biological samples from a tertiary hospital in the Northeast region, Brazil.

\section{METHODS}

We analyzed 596 health care professionals at Hospital das Clínicas, Universidade Federal de Pernambuco (HC-UFPE). Blood samples were obtained from October to November 2020 for the measurement of serum vitamin D, which was performed using the Architect 25-OH Vitamin D Kit (Abbott Diagnostics, United States), using the chemiluminescent microparticle immunoassay methodology. Data related to participants, i.e., sex and age were obtained. In parallel with the dosage of vitamin D, we also performed tests for SARSCoV-2 infection through qualitative immunochromatographic tests for the detection of immunoglobulin $\mathrm{G}(\mathrm{IgG})$ antibodies against the virus (COVID-19 IgG ECO test-ECO Diagnóstica, Brazil).

As recommended by the consensus of the Brazilian Society of Clinical Pathology/Laboratory Medicine and Brazilian Society of Endocrinology and Metabolism, the reference values for vitamin $\mathrm{D}$ were stratified according to age and clinical conditions: $>20 \mathrm{ng} / \mathrm{mL}$, a desirable value for a healthy population (up to 60 years of age); between 30 and $60 \mathrm{ng} / \mathrm{mL}$, the recommended value for at-risk groups such as elderly people, pregnant women, breastfeeding women, patients with syndromes related levels of vitamin D; and above $100 \mathrm{ng} / \mathrm{mL}$, the risk of toxicity and hypercalcemia ${ }^{7}$.

Descriptive statistics were revised for the variables. A comparison was made between vitamin $\mathrm{D}$ levels and serological reactivity with SARS-CoV-2-IgG. The Fisher's exact test was used for qualitative variables and the Student's $t$-test for

\footnotetext{
${ }^{1}$ Universidade Federal de Pernambuco, Hospital das Clínicas - Recife (PE), Brazil.

${ }^{2}$ Universidade de Pernambuco, Institute of Biological Sciences - Recife (PE), Brazil.

${ }^{3}$ Universidade Federal de Pernambuco, Medical Sciences Center, Postgraduation in Tropical Medicine - Recife (PE), Brazil.

${ }^{4}$ Faculdade Pernambucana de Saúde, Instituto de Medicina Integral Professor Fernando Figueira - Recife (PE), Brazil.

*Corresponding author: kledoaldo@gmail.com

Conflicts of interest: the authors declare there are no conflicts of interest. Funding: none.

Received on July 29, 2021. Accepted on August 01, 2021.
} 
continuous variables. The normal distribution of continuous variables was verified using the Shapiro-Wilk normality test. The differences were considered statistically significant when $\mathrm{p}<0.05$. All statistical analyses were performed using SPSS Statistics software, version 25 for Windows (IBM Corporation, United States). This study was approved by the Ethics Committee of the Health Sciences Centre of Universidade Federal de Pernambuco on October 16, 2020 with protocol number 38060620.6.0000.5208.

\section{RESULTS}

Out of the 596 health care professionals who worked directly with patients diagnosed with COVID-19, the majority were females $(\mathrm{n}=451,76 \%)$, and a high serological reactivity for SARS-CoV-2 IgG ( $\mathrm{n}=250,42 \%)$ was detected. The serum dosage of vitamin $\mathrm{D}$ was carried out in 571 health care workers, out of which 492 (86.2\%) health care workers had levels considered desirable ( $>20 \mathrm{ng} / \mathrm{mL}$ ). Our findings demonstrate an association between vitamin D insufficiency $(\leq 20 \mathrm{ng} / \mathrm{mL})$ in females and people under the age of 50 years $(\mathrm{p}<0.001)$ (Table 1). There was a statistical association between serological reactivity for SARS-CoV-2 and health care workers over 50 years old $(\mathrm{p}<0.001)$ (Table 2$)$. Regarding the relationship between serum vitamin $D$ levels and the presence of $\operatorname{IgG}$ antibodies to SARS-CoV-2 in the asymptomatic health care professionals evaluated, we found that there was an inverse relationship between the serological reactivity to the virus and the levels of vitamin $\mathrm{D}$, that is, health care professionals who were tested positive for SARS-CoV-2 had higher serum vitamin D levels.

Table 1. Characteristics of health care professionals related to the concentration of serum levels of vitamin $D$.

\begin{tabular}{l|c|c|c}
\multirow{2}{*}{} & \multicolumn{3}{c}{ Vitamin D } \\
\cline { 2 - 4 } Sex (n=569) & $\leq 20 \mathrm{ng} / \mathrm{mL}$ & $>20 \mathrm{ng} / \mathrm{mL}$ & p-value \\
\hline Female & 58 & 374 & $<0.001$ \\
\hline Male & 21 & 116 & \\
\hline Age (years) (n=569) & 76 & 403 & $<0.001$ \\
\hline$\leq 50$ & 08 & 82 & \\
\hline $51-64$ & 28 & 211 & $<0.001$ \\
\hline Point of care test IgG COVID-19 (n=571) & \\
\hline Reagent & 51 & 281 & \\
\hline Non-reagent & \multicolumn{3}{|c}{} \\
\hline
\end{tabular}

IgG: immunoglobulin G; COVID-19: coronavirus disease 2019.

\section{DISCUSSION}

We found that there was an inverse relationship between the serological reactivity to the virus and the levels of vitamin D, that is, health care professionals who were tested positive for SARS-CoV-2 had higher serum vitamin D levels. This result is the opposite of that observed by Meltzer et al. and Kaufman et al. in individuals in the United States ${ }^{4,6}$. Meanwhile, Hastie et al. showed no statistical association between serum vitamin $\mathrm{D}$ levels and serological reactivity to SARS-CoV-2 in individuals tested in the United Kingdom ${ }^{2}$. In addition, Murai et al. demonstrated, in a clinical trial conducted in Brazil with patients admitted to a hospital in a moderate or severe state of COVID-19, that a single dose of 200,000 IU of vitamin D did not interfere in mortality, length of stay in the hospital, the number of admissions to the intensive care unit, or the need for mechanical ventilation when compared with placebo ${ }^{8}$.

Our findings demonstrate an association between vitamin D insufficiency $(\leq 20 \mathrm{ng} / \mathrm{mL})$ in females and people under the age of 50 years. Meltzer et al. also showed an association of lower serum vitamin D levels and the age of $<50$ years ${ }^{6}$; however, the same was not evidenced for females. There was a statistical association between serological reactivity for SARS-CoV-2 and health care workers over 50 years old $(\mathrm{p}<0.001)$ (Table 2). This result is important, as it illustrates a greater susceptibility of older health care professionals to viral infection, which causes greater concern since it is known that older individuals may be more prone to more severe symptoms of the disease.

One of the limitations of this research was the fact that, although the individuals evaluated were asymptomatic in the period of serological diagnosis, there were no data available regarding the results of RT-PCR tests for SARS-CoV-2 in these professionals in the months before the research. Due to the less data available about the action of vitamin D in COVID-19 and some of them have conflicting results or have different study designs and populations,

Table 2. Characteristics of health care professionals related to the serological results of IgG for SARS-CoV-2.

\begin{tabular}{|c|c|c|c|}
\hline & \multicolumn{3}{|c|}{ Rapid test IgG COVID-19 } \\
\hline & Reagent & Non-reagent & p-value \\
\hline \multicolumn{4}{|c|}{ Sex $(n=594)$} \\
\hline Female & 190 & 261 & 1.00 \\
\hline Male & 59 & 84 & \\
\hline \multicolumn{4}{|c|}{ Age (years) $(n=578)$} \\
\hline$\leq 50$ & 195 & 288 & $<0.001$ \\
\hline $51-64$ & 47 & 48 & \\
\hline
\end{tabular}

IgG: immunoglobulin G; SARS-CoV-2: severe acute respiratory syndrome coronavirus 2; COVID-19: coronavirus disease 2019. 
it is necessary to carry out studies that are better designed to evaluate the role of vitamin $\mathrm{D}$ in preventing viral infection.

\section{CONCLUSIONS}

Our results demonstrated an association between low serum vitamin D concentrations and nonreactive serological testing for viral infection. However, it appears that there are conflicting results in the literature on the role of vitamin D in decreasing the chances of infection or progression to more severe symptoms by SARS$\mathrm{CoV}-2$. Thus, other clinical trials are required to understand the real role of vitamin $\mathrm{D}$ supplementation, either in preventing infection or decreasing the progression to more severe symptoms.

\section{ACKNOWLEDGMENTS}

We would like to thank all the health care professionals who agreed to participate in the research at this very delicate moment in public health worldwide and the laboratory analysts for carrying out the tests.

\section{AUTHORS" CONTRIBUTIONS}

ACL: Data curation, Formal analysis, Investigation, Methodology, Writing - original draft. APS: Data curation, Formal analysis, Investigation, Methodology, Writing - original draft. MVD: Data curation, Formal analysis, Investigation, Methodology, Writing original draft. AVN: Data curation, Formal analysis, Investigation, Methodology, Writing - original draft. WRCS: Data curation, Formal analysis, Investigation, Methodology, Writing - original draft. CLDP: Data curation, Formal analysis, Investigation, Methodology, Writing - original draft. MLR: Data curation, Formal analysis, Investigation, Methodology, Writing-original draft. EBCJ: Investigation, Methodology, Writing - review \& editing. KL: Conceptualization, Data curation, Formal analysis, Investigation, Methodology, Writing - original draft, Writing - review \& editing.

\section{REFERENCES}

1. Honardoost M, Janani L, Aghili R, Emami Z, Khamseh ME. The association between presence of comorbidities and covid-19 severity: a systematic review and metaanalysis. Cerebrovasc Dis. 2021;50(2):132-40. https://doi. org/10.1159/000513288

2. Hastie CE, Mackay DF, Ho F, Celis-Morales CA, Katikireddi $S V$, Niedzwiedz CL, et al. Vitamin D concentrations and COVID-19 infection in UK Biobank. Diabetes Metab Syndr. 2020;14(4):561-5. https://doi.org/10.1016/j.dsx.2020.04.050

3. Maghbooli Z, Sahraian MA, Ebrahimi M, Pazoki M, Kafan S, Tabriz $H M$, et al. Vitamin D sufficiency, a serum 25-hydroxyvitamin D at least $30 \mathrm{ng} / \mathrm{mL}$ reduced risk for adverse clinical outcomes in patients with COVID-19 infection. PLoS One. 2020;15(9):e0239799. https://doi.org/10.1371/journal.pone.0239799

4. Kaufman HW, Niles JK, Kroll MH, Bi C, Holick MF. SARS-CoV-2 positivity rates associated with circulating 25-hydroxyvitamin D levels. PLoS One. 2020;15(9):e0239252. https://doi. org/10.1371/journal.pone.0239252

5. Dancer RC, Parekh D, Lax S, D'Souza V, Zheng S, Bassford CR, et al. Vitamin $D$ deficiency contributes directly to the acute respiratory distress syndrome (ARDS). Thorax. 2015;70(7):61724. https://doi.org/10.1136/thoraxjnl-2014-206680

6. Meltzer DO, Best TJ, Zhang H, Vokes T, Arora V, Solway J. Association of Vitamin D status and other clinical characteristics with COVID-19 test results. JAMA Netw Open. 2020;3(9):e2019722. https://doi.org/10.1001/ jamanetworkopen.2020.19722

7. Ferreira CES, Maeda SS, Batista MC, Lazaretti-Castro $M$, Vasconcellos LS, Madeira $M$, et al. Consensus reference ranges of vitamin $\mathrm{D}[25(\mathrm{OH}) \mathrm{D}]$ from the Brazilian medical societies. Brazilian Society of Clinical Pathology/ Laboratory Medicine (SBPC/ML) and Brazilian Society of Endocrinology and Metabolism (SBEM). J Bras Patol Med Lab. 2017;53(6):377-81. https://doi.org/10.5935/16762444.20170060

8. Murai IH, Fernandes AL, Sales LP, Pinto AJ, Goessler KF, Duran CSC, et al. Effect of a single high dose of vitamin d3 on hospital length of stay in patients with moderate to severe COVID-19: a randomized clinical trial. JAMA. 2021;325(11):1053-60. https://doi.org/10.1001/jama.2020.26848 